\title{
RBF Network-Based Chaotic Time Series Prediction and It's Application in Foreign Exchange Market
}

\author{
Lili Ma Xusong Xu \\ School of Economics and Management, Wuhan University, Wuhan 430072, P. R. China
}

\begin{abstract}
The foreign exchange market is a chaotic dynamic system. We apply the RBF network-based chaotic time series prediction on the daily USD/RMB exchange rate. We apply the RBF network and phase space reconstruction to find the optimal embedding dimension in the foreign exchange market from the point view of forecasting. We find that the optimal embedding dimension is 10 . As a result the dimension of the attractor of the market is about in the interval between 4 and 5. Finally, we use the optimal embedding dimension to implement the prediction.
\end{abstract}

Keywords: Chaotic time series, Prediction, Phase space reconstruction, RBF network, Foreign exchange market

\section{Introduction}

A chaotic system is a relatively complex behavior through the deterministic dynamics of nonlinear systems. Deterministic laws make the orbits of the system attracted to a complex higher-dimensional subset called a strange attractor. The chaotic system is sensitivity to initial conditions; points that are arbitrarily close initially become exponentially further apart with increasing time, leading to the amplification of very small perturbations into global uncertainties. As a result it is not possible to make accurate longterm predictions. The importance of studying chaotic behavior lies in the fact that chaotic behavior is much more widespread, and may even be the norm in the real world. Scheindman and Lebaron, Frank and Stengos found the chaotic behavior in financial market such as stock market, foreign exchange markets and futures market [1]-[2]. Chaotic time series prediction becomes an extremely important research area and obtains widespread application [3]-[4]. The phase space reconstruction and embedding theorem proposed by Takens supply the theory foundation for nonlinear time series prediction [5]. In the phase space reconstructed artificial neural networks can deal with the mapping relations between the current situation and future situation to implement the chaotic time series prediction.

BP (back propagation) neural network is universally applied in nonlinear prediction. Since BP neural network encounters local minimum, slow convergence speed and convergence instability, the shortcomings should be overcome by application of other new method. Radial basis function network is a special type of feed-forward neural network. It has received considerable attention recently due to its universal approximation properties and simple parameter estimation in the field of interpolation regression and classification [6]-[7]. Due to its nonlinear approximation properties, RBF network is able to fit the training data in multidimensional space. As a direct consequence, RBF's properties made them attractive for complex prediction in nonlinear dynamics systems of financial market [8].

In this paper we will apply the RBF networkbased chaotic time series prediction to the foreign exchange market in China. The paper is structured as follows: in section 2 we describe the method of chaotic time series prediction based on RBF network. In section 3 we apply the method to the foreign exchange market, and obtain the optimal embedding dimension in China exchange market. Section 4 gives the conclusion.

\section{Chaotic time series prediction based on RBF network}

We can not exactly know the attractors of the real dynamic system in foreign exchange market. We can only observe the exchange rate series, which are unable to exhibit the inherent essential character of the dynamic system. But in fact, the time series actually imply the information and characteristic of other variables even the whole system, because the evolvement of any subset in the system is determined by all other variables. The phase space reconstruction can embed one-dimensional time series in higherdimensional phase space, and the attractor in the original phase space is completely unfolded in the reconstructed phase space. If a time series comes from 
a dynamic system that is on an attractor, the trajectories constructed from the time series by embedding will have the same topological properties as the original one. That is to say phase space reconstruction enables us to study unobserved variables. In reconstructed space we can map the relation between current and future situation with artificial neural networks.

\subsection{Radial basis function networks}

A standard radial basis function networks are embedded into a two-layer feed-forward neural network. The network is characterized by a set of inputs and a set of outputs. Between the inputs and outputs there is a layer of processing units called hidden units, each of which implements a radial basis function. The nodes in the hidden layer operate on the distance from an applied input vector to an internal parameter vector, called a center. The response of each basis function is a nonlinear function of the distance and is radically symmetric about the center. Various functions have been tested as activation functions for RBF network. In this paper we use the Gaussian activation function. The output layer implements a weighted sum of hidden-unit outputs. The mapping function is given by:

$$
p_{j}(X)=\sum_{i=1}^{n} w_{i j} \exp \left[-\left(X-\mu_{j}\right)^{T} \Sigma_{j}^{-1}\left(X-\mu_{j}\right)\right] \text {, }
$$

for $j=1,2, \cdots l$, where $X \in R^{N}$ is input $N$ dimensional vector, and $p_{j}(X)$ is the output of the $j$ th units. $w_{i j}$ are the output weights, each corresponding to the connection between a hidden unit $i$ and an output unit $j$ and $n$ represents the number of output units. $\mu_{j}$ and $\Sigma_{j}$ are the mean and the covatiance matrix of the $j$ th Gaussian function. Geometrically, a radial basis function represents a bump in the multidimensional space. In order to model such a mapping we have to find the centers of Gaussian function, output weights and the number of hidden units. Meanwhile we must design the structure of the network to improve the generalization performance.

\subsection{Phase space reconstruction}

Phase space reconstruction was firstly proposed by Packard [9] and was proved by Takens. Let $M$ be a compact manifold of dimension $\mathrm{d}$, and the dynamic system is defined as $\partial y / \partial t=G(y)$, where $\partial$ represents differential operator. $G(\cdot): M \rightarrow M$ is a smooth mapping. Let $y_{0} \in M$, there is only solution $y\left(t, y_{0}\right)$ in dynamic system $\partial y / \partial t=G(y)$ passing through $y_{0}$. Let $\phi_{t}\left(y_{0}\right)=y\left(t, y_{0}\right)$. We define $\phi_{t}: M \rightarrow M, \forall t \in R$, and obviously $\phi_{t}$ satisfies:

(1) $\phi_{0}(y)=y, \quad \forall y \in M$;

(2) $\phi_{t}\left(\phi_{s}(y)\right)=\phi_{t+s}(y), \forall y \in M, \forall t, s \in R$.

Take any time series $\left\{\phi_{n T}\left(Z_{0}\right), Z_{0} \in M\right.$, $n=0,1,2, \cdots$,$\} in the compact manifold, note f=\phi_{t}$, and the time series can be written as $\left\{f^{n}\left(Z_{0}\right)\right.$, $\left.Z_{0} \in M, n=0,1, \cdots,\right\}$, or $\left\{Z_{n}, n \in N\right\}$, where $Z_{n+1}=f\left(Z_{n}\right)$. Let $h: M \rightarrow R$ is a observe function, we can write down the observation time series $\left\{x_{n}\right\}$, where $x_{n}=h\left(f^{n}\left(Z_{0}\right)\right)=h\left(f\left(f^{n-1}\left(Z_{0}\right)\right)\right)$. Define mapping $\varphi: M \rightarrow R^{m} \quad$, satisfying $\varphi(Z)=$ $\left(h(Z), h\left(f^{\tau}(Z)\right), \cdots h\left(f^{(m-1) \tau}(Z)\right)\right)$, where $m$ is the embedding dimension, and $\tau$ is the time delay. Delay vectors $X_{n}$ can be written as $X_{n}=\varphi\left(Z_{n}\right)=$ $\left(x_{n}, x_{n-\tau}, \cdots x_{n-(m-1) \tau}\right)$.

Takens [5] proved that if the curve is in the compact regions of the phase space and the embedding dimension satisfy $m \geq 2 d+1$, and then $\varphi$ is a one to one mapping. The embedding $\varphi$ maps from $M$ to $R^{m}$, and then we can analyze the structure of the trajectory of the dynamic system in $R^{m}$ and easily infer properties of the actual trajectory on the attractor in $M$. Define a composite function $F=$ $\varphi \circ f \circ \varphi^{-1}: R^{m} \rightarrow R^{m}$, which satisfies: $F\left(X_{n}\right)=$ $\varphi \circ f \circ \varphi^{-1}\left(X_{n}\right)=\varphi \circ f\left(Z_{n}\right)=\varphi\left(Z_{n+1}\right)=X_{n+1}$. So if $h$ and $f$ are continuous and differential, then so is $\varphi$, which means the trajectories constructed from the time series by embedding will have the same topological properties as the original dynamic system.

\subsection{Chaotic time series prediction}

If we find the embedding dimension $m$, according to the method of phase space reconstruction, we can reconstruct an m-dimensional phase space from the time series $\left\{p_{t}\right\}$. Let the vector of the phase space marked as $P_{t}=\left(p_{t}, p_{t-\tau}, p_{t-2 \tau}, \cdots, p_{t-(m-1) \tau}\right)$. And there exists a smooth function $F: R^{m} \rightarrow R^{m}$, satisfying $F\left(P_{t}\right)=P_{t+1}$. We can infer from $F$ a 
function $f: R^{m} \rightarrow R$, satisfying $p_{t+1}=f\left(P_{t}\right)$. As a result choosing the embedding dimension $m$ is very important, with which we can predict $p_{t+1}$ from the history data. Takens considered that the sufficient condition for embedding dimension is $m \geq 2 d+1$. However, too large embedding dimension needs more observations and complex computation. If $\mathrm{m}$ is too large, chaotic data add redundancy and degrade the performance of many algorithms. It is more difficult to separate the deterministic system from the signal noises in observations [10]. As a result if the original attractor has dimension $\mathrm{d}$, then an embedding dimension of $\mathrm{m}=2 \mathrm{~d}+1$ will be adequate for reconstructing the attractor.

We apply RBF network to find the optimal embedding dimension. We construct a RBF network to fit the nonlinear function $\hat{f}$, which can approximate $f$ precisely. Take $p_{t+1}=\hat{f}\left(p_{t}, p_{t-\tau}, \cdots, p_{t-(m-1) \tau}\right)$ $+\varepsilon_{t}$. Through the training of the network with the sample, we can obtain different $\varepsilon$ with different dimension $m$. The noise $\varepsilon$ will diminish with $m$ increases. Then we can obtain the optimal $m$ when $\varepsilon_{t}$ is minimal.

\section{Application}

We apply the chaotic time series prediction based on RBF network to the foreign exchange market in China. We take the U.S dollar as basic exchange rate. In the following empirical studies, we use the daily USD/RMB rate from 7-22-2005 to 3-20-2007. We take two steps. In the first step, we apply the RBF network and phase space reconstruction to find the optimal embedding dimension in the foreign exchange market. In the second step, we use the optimal embedding dimension to implement the prediction.

\subsection{Optimal embedding dimension}

Let $\left\{p_{t}\right\}$ represents the observable time series of exchange rate with $t=1,2, \cdots, \mathrm{N}$, and the time delay $\tau$ is 1 . According to the phase space reconstruction, we construct from the original time series $p_{t}$ vectors of dimension $m, P_{t}=$ $\left(p_{t}, p_{t-1}, p_{t-2}, \cdots, p_{t-(m-1)}\right) \cdot p_{t+1}=\hat{f}\left(P_{t}\right)$ describe the relation between the current state and future rate. We take the $\left\{\left(P_{t}, p_{t+1}\right): t=1,2, \cdots, N-(m-1)\right\}$ as the training data, and apply RBF network to simulate the exchange rate. In our RBF network, there are $m$ hidden units and one output unit. Vector $P_{t}$ is the inputs, and $\hat{f}$ is the output. The radial basis function in hidden units implement nonlinear computations to inputs and the output layer implements a weighted sum of hidden-unit outputs. We compare the output $\hat{f}$ with the target output $p_{t+1}$, and define the mean square error as:

$$
M S E=\left(\hat{f}\left(P_{t}\right)-p_{t+1}\right)^{2} /(N-(m-1))
$$

With different embedding dimension $m$, we can obtain different mean square errors. When dimension $m$ is optimal when MSE gets minimal. We picture the MSE with different embedding dimension $\mathrm{m}$ in Fig. 1. From the figure it is obviously that the optimal embedding dimension is 10 when we apply the prediction with RBF network. According to the embedding theorem, $m \geq 2 d+1$, we can know that the dimension of the attractor is between 4 and 5 in foreign exchange market.

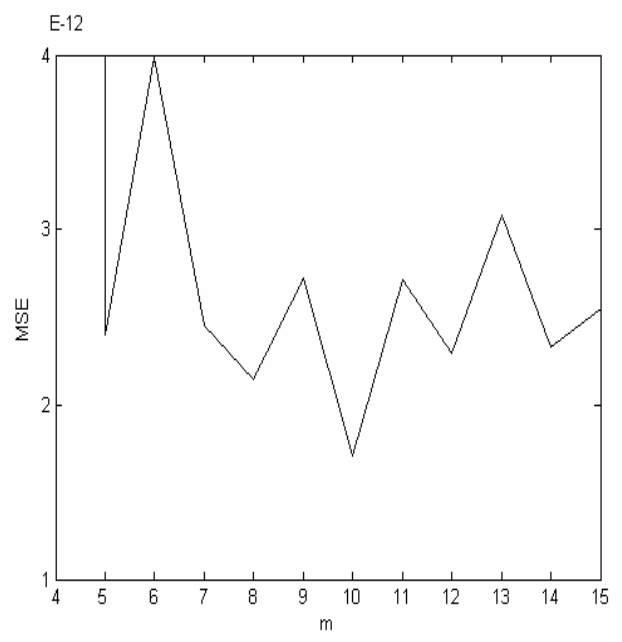

Fig. 1: MSE-m relationship. This shows the MSE with different embedding dimensions.

\subsection{Prediction with optimal embedding dimension}

From the Fig.1, it is obviously that the optimal embedding dimension is 10 . We can make a conclusion that when we take $m=10$, the error of the prediction must be minimal. With the same sample, we train the RBF network. The training error is depicted in Fig. 2. We use the basic exchange rate from 3-21-2007 to 4-11-2007 to test prediction effect. And the results are in Table 1 . The forecasting results are measured by the mean squared error, which is 0.0345 . 


\begin{tabular}{|c|c|c|c|}
\hline Date & $3-21-2007$ & $3-22-2007$ & $3-23-2007$ \\
\hline $\begin{array}{c}\text { USD/RMB } \\
\text { exchange rate }\end{array}$ & 7.7355 & 7.731 & 7.7359 \\
\hline $\begin{array}{c}\text { Estimation with } \\
\text { RBF network }\end{array}$ & 7.7743 & 7.663 & 7.7364 \\
\hline Date & $3-26-2007$ & $3-27-2007$ & $3-28-2007$ \\
\hline $\begin{array}{c}\text { USD/RMB } \\
\text { exchange rate }\end{array}$ & 7.7393 & 7.7343 & 7.7318 \\
\hline $\begin{array}{c}\text { Estimation with } \\
\text { RBF network }\end{array}$ & 7.728 & 7.7016 & 7.7089 \\
\hline Date & $3-29-2007$ & $3-30-2007$ & $4-2-2007$ \\
\hline $\begin{array}{c}\text { USD/RMB } \\
\text { exchange rate }\end{array}$ & 7.7303 & 7.734 & 7.731 \\
\hline $\begin{array}{c}\text { Estimation with } \\
\text { RBF network }\end{array}$ & 7.7313 & 7.667 & 7.747 \\
\hline Date & $4-3-2007$ & $4-4-2007$ & $4-5-2007$ \\
\hline $\begin{array}{c}\text { USD/RMB } \\
\text { exchange rate }\end{array}$ & 7.7277 & 7.7349 & 7.7268 \\
\hline $\begin{array}{c}\text { Estimation with } \\
\text { RBF network }\end{array}$ & 7.6898 & 7.7337 & 7.7103 \\
\hline
\end{tabular}

Table 1: Prediction with $\mathrm{m}=10$.

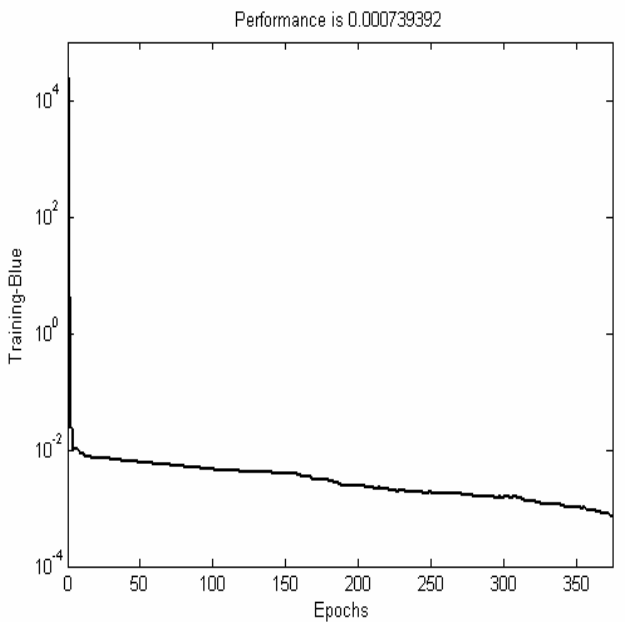

Fig. 2: Training error. This shows the training error with epochs increase.

\section{Conclusions}

The phase space reconstruction and embedding theorem proposed by Takens supply the theory foundation for nonlinear time series prediction. In the reconstructed phase space artificial neural networks can deal with the mapping to implement the chaotic time series prediction. Radial basis function network is a special type of feed-forward neural network. It has nonlinear approximation properties to fit the training data in higher-dimensional space and model complex mappings. In this paper we apply the RBF network and phase space reconstruction on daily USD/RMB exchange rate, and find that the optimal embedding dimension is 10. As a result the dimension of the attractor of foreign exchange market is about in the interval between 4 and 5 . Therefore, the foreign exchange market is a chaotic system, and we must take the nonlinear analytical instruments. Finally, we use the optimal embedding dimension to implement the prediction.

\section{Acknowledgement}

This work is partially supported by National Nature Science Foundation of China (Grant No. 70440003).

\section{References}

[1] J.A. Scheindman, B. Lebaron, Nonlinear dynamics and stock returns. The Journal of Business, 62: 311-337, 1989.

[2] M.Z. Frank, T. Stengos, Some evidence concerning macroeconomic chaos. Journal of Monetary Economics, 22: 423-438, 1988.

[3] W. Shangfei, Z. Peiling, Application of radial basis function neural network to stock forecasting. Forecasting, 6: 44-46, 1998.

[4] X.S. Xu, B.P. Xiong, The fractal dimension of shanghai stock market based on RBF neural networks. Wuhan University Journal (Natural Science Edition), 49: 309-312, 2003.

[5] F. Takens, Detection strange attractor in turbulence. Lecture Notes in Math, 898: 366-381, 1981.

[6] M. Burger, A. Neubauer, Error bounds for approximation with neural networks. Journal of Approximate Theory, 2: 235-250, 2001.

[7] A. Krzyzak, T. Linder, C. Lugosi, Nonparametric estimation and classification using radial basis function nets and empirical risk minimization. IEEE Trans. on Neural Networks, pp. 475-487, 1996.

[8] D.Y. Ye, W.B. Liu, Application of radial basis function neural network to pattern classification of stock changing tendency. Operations Research and Management Science, 8: 46-50, 1999.

[9] N.H. Packard, J.P. Crutchfield, J.D. Farmer and R.S. Shaw, Geometry from a time series, Physical Reviews Letters. 45: 712-716, 1980.

[10] H. Kantz, T. Schreiber, Nonlinear time series analysis, Cambridge University Press, pp. 34-37, 1977. 